\title{
A volatilidade de projetos industriais para uso em análise de risco de investimentos
}

\section{The volatility of industrial projects for use in analysis of risk in investments}

\author{
Ronildo Jorge de Oliveira ${ }^{1}$ \\ Edson de Oliveira Pamplona ${ }^{1}$
}

\begin{abstract}
Resumo: A volatilidade em projetos industriais é um parâmetro significativo na análise de risco de investimentos. Quando se avaliam ativos financeiros, a volatilidade pode ser determinada por meio de dados históricos do ativo, porém, ao se trabalhar com projetos industriais, normalmente, não existem dados históricos. Este fato torna a volatilidade um parâmetro crítico de ser determinado. Este artigo faz a aplicação e a comparação de três abordagens para determinação da volatilidade de um projeto de uma indústria de transformação. Duas destas abordagens adotadas, a "Abordagem Consolidada da Volatilidade de Copeland e Antikarov (2001)" e a "Estimativa de Volatilidade de Herath e Park (2002)", foram obtidas a partir de uma revisão bibliográfica de métodos para cálculo de volatilidade de projetos de investimentos. A terceira abordagem é uma nova proposta para o cálculo da volatilidade, chamada de "A Estimativa da Volatilidade Através da Dependência entre VP e VP," . O trabalho realiza a avaliação tradicional de investimentos em um projeto industrial, no qual são aplicadas as três diferentes abordagens de determinação da volatilidade. Conclui-se, com um comparativo entre as três abordagens, que a diferença entre o maior e o menor valor encontrado para a volatilidade é de dezenove pontos percentuais.
\end{abstract}

Palavras-chave: Volatilidade. Risco. Opções reais. Análise de investimento.

\begin{abstract}
The volatility concerning industrial projects is a significant parameter in the analysis of risk in investments. When financial assets are evaluated, the volatility can be determined by historical data of assets. However, when working with industrial projects, usually, there are no historical data, which makes volatility a critical parameter to be determined. The objective of this study is the adoption and comparison of three approaches to determine project volatility in a transformation industry. Two of the adopted approaches, the "Consolidated Volatility Approach of Copeland and Antikarov (2001)" and the "Estimated Volatility of Herath and Park (2002)" were obtained from a literature review of methods for calculation of volatility of investment projects. The third approach is a new proposal for volatility calculation entitled: "The Estimate of Volatility through the Dependence between $P V_{o}$ and $P V_{1}$ ”. A traditional analysis of investments in an industrial project was performed, in which these three different approaches for determination of volatility were adopted. Lastly, a comparison of the three approaches is presented, in which the difference between the highest and the lowest volatility values is nineteen percentile points.
\end{abstract}

Keywords: Volatility. Risk. Real options. Investment analysis.

\section{Introdução}

\subsection{Considerações iniciais}

Os avanços tecnológicos ocorridos nas últimas décadas têm contribuído para que, de forma exponencial, as empresas busquem técnicas de análise de investimentos mais sofisticadas. Estas novas técnicas buscam ser mais precisas e considerar algumas variáveis que as técnicas tradicionais não consideram. A utilização de técnicas mais precisas e que consideram mais variáveis contribuem para uma maior assertividade por parte dos gestores no momento decisivo para a realização ou não de um projeto. Este fato evita que as empresas subavaliem seus investimentos ou realizem investimentos que não sejam rentáveis ou, ainda, abandonem investimentos que poderiam ser rentáveis.

Os métodos de análise de investimentos que têm como base o Fluxo de Caixa Descontado (FCD) são

\footnotetext{
${ }^{1}$ Instituto de Engenharia de Produção e Gestão, Universidade Federal de Itajubá - UNIFEI, CP 50, CEP 37500-903, Itajubá, MG, e-mail: ronildooliveira@terra.com.br; pamplona@unifei.edu.br
} 
largamente utilizados nas indústrias para análise de viabilidade ou não de um projeto, porém é crescente a necessidade do uso de técnicas que abordem a flexibilidade gerencial que a maioria dos projetos possui. Uma técnica que é uma vertente das Opções Financeiras e que ficou conhecida como Teoria das Opções Reais, busca incorporar as flexibilidades gerenciais que os métodos tradicionais não incorporam. A Teoria das Opções Financeiras surgiu na década de 70, com a publicação do trabalho de Black e Scholes (1973). Nas décadas seguintes, outras publicações corroboraram com trabalho destes autores, aperfeiçoando o modelo desenvolvido por eles para o uso em ativos fixos.

Miranda Filho (2005), sobre a expansão do sistema elétrico, afirma que a introdução do pensamento de Opções no planejamento da expansão deste sistema vem ao encontro das necessidades dos executivos de uma ferramenta que lhes permita considerar cientificamente as opções de adiamento, expansão, abandono e contratação no mercado consumidor de energia elétrica.

Seguindo o pensamento de Miranda, na área industrial, o planejamento de construção de novas unidades e, até mesmo, o planejamento de expansões, utilizando a Teoria das Opções Reais, atende às necessidades dos executivos de uma ferramenta que lhes permita maior flexibilidade, considerando fatores que os métodos tradicionais não consideram, como o adiamento, o investimento em diferentes datas e o abandono de projetos. Sendo assim, as empresas devem sofisticar-se na maneira como avaliam seus investimentos, utilizando ferramentas complementares aos métodos tradicionais, capazes de captar fatores que eles não levam em consideração, auxiliando assim na tomada de decisão.

Conforme enfatizado por Luehrman (1998), a maioria dos executivos questiona: "Como usar a precificação de opções em um projeto, com números reais, em vez de exemplos estéreis?"; também, Santos e Pamplona (2005) afirmam que: "embora muito se tenha dito a respeito do poder da teoria das opções reais aplicada à análise de investimento, o Brasil ainda carece de trabalhos que venham a comprovar sua validade. É necessário que esta teoria seja cada vez mais difundida, principalmente no meio corporativo." Baseados em entrevistas realizadas com executivos de diversas empresas, Triantis e Borison (2001) concluem que a Teoria das Opções Reais tem ajudado aos gerentes a tomar melhores decisões nas análises de investimentos.

Conforme destacado por Brandão et al. (2005a), para o uso da abordagem das opções reais, é necessário conhecer apenas três parâmetros do projeto sob estudo, sendo estes:

i. a estimativa do valor presente do projeto;

ii. a volatilidade dos retornos do projeto;

iii. a taxa livre de risco.
A estimativa do valor presente do projeto pode ser obtida com o uso do Fluxo de Caixa Descontado; como taxa livre de risco podem ser utilizados, por exemplo, os retornos de um título do governo; porém a determinação da volatilidade não é tarefa tão simples como dos demais parâmetros.

Do exposto, este trabalho busca estimar a volatilidade em um projeto industrial a partir de modelos obtidos em revisão bibliográfica das diversas abordagens de determinação da volatilidade para o uso em ativos fixos, como também, a partir de um novo modelo proposto. Os resultados serão comparados. A volatilidade é um dos componentes mais relevantes na avaliação do valor das opções em investimentos com risco.

\subsection{Objetivo}

O objetivo deste trabalho é apresentar uma nova abordagem para o cálculo da volatilidade a partir da adaptação de sistemáticas existentes, comparando os resultados por meio de sua aplicação em um projeto industrial.

\subsection{Metodologia}

O método de pesquisa a ser utilizado no presente trabalho será a MODELAGEM E SIMULAÇÃO. Tal método de pesquisa tem as características apresentadas por Bertrand e Fransoo (2002). Será realizada uma análise comparativa entre os principais modelos existentes, destacando as principais diferenças entre eles.

\section{Volatilidade}

A volatilidade é um parâmetro de entrada significativo na abordagem de opções, tanto financeiras, quanto de ativos reais. No trabalho com opções financeiras, a volatilidade é um parâmetro que pode ser estimado pelos dados históricos, o que torna o resultado das opções muito próximo da realidade. No trabalho com ativos reais, na maioria dos casos, não se trabalha com dados históricos para se estimar a volatilidade, o que, se não for feito de forma criteriosa, pode levar a uma sub ou superestimativa da volatilidade, levando o cálculo das opções a um valor distante do valor real.

Nestes casos, em que não se trabalha com dados históricos, uma alternativa é a de se utilizar simulação de Monte Carlo para calcular a estimativa da volatilidade dos fluxos de caixa. No método de Monte Carlo, as principais fontes de incerteza do projeto, tais como: receitas, taxas de desconto, custos e despesas, impostos e depreciação, entre outras, podem servir como variáveis de entrada para a simulação.

Alguns autores, nos anos recentes, têm trabalhado nesta árdua tarefa de propor modelos para estimar 
a volatilidade para o trabalho com ativos reais, sugerindo diferentes variações para a aplicação da simulação de Monte Carlo. Nos modelos propostos, a volatilidade do ativo subjacente é estimada pelo cálculo do desvio padrão da taxa de retorno, que, por sua vez, é desenvolvida por meio de uma distribuição de probabilidades, que é simulada por meio do método de Monte Carlo.

A Abordagem Consolidada da Incerteza baseada no Valor Presente Logarítmico de Copeland e Antikarov (2001) e Herath e Park (2002), o Valor Presente Logarítmico Condicional de Brandão et al. (2005b), que foi comentado por Smith (2005), são publicações recentes que sugerem a aplicação da simulação de Monte Carlo para a estimativa da volatilidade.

\subsection{O Movimento Geométrico Browniano}

Os trabalhos de Copeland e Antikarov (2001), Herath e Park (2002) e de Brandão et al., (2005b), além de utilizarem a simulação de Monte Carlo, têm em comum a hipótese de considerar que o valor do ativo subjacente segue o Movimento Geométrico Browniano (MGB), ou seja, o ativo subjacente nunca pode ter valores negativos, sendo logarítmica normal (lognormal) a distribuição do valor final.

Isto implica dizer que a variação no valor do retorno do projeto no tempo segue um caminho aleatório (random walk).

Conforme destacado por Brandão et al. (2005b), se $\mathrm{V}_{\mathrm{i}}$ e $\mathrm{V}_{\mathrm{i}+1}$ representarem, sem pagamento de dividendos, o valor de um projeto nos períodos " $\mathrm{i}$ " e "i+1", o retorno em tempo contínuo $\left(\ln \left(\mathrm{V}_{\mathrm{i}+1} / \mathrm{V}_{\mathrm{i}}\right)\right)$, pela hipótese do caminho aleatório, tem uma distribuição normal com média " $v$ " e desvio padrão " $\sigma$ ".

No regime de capitalização contínua, os valores "fluem contínua e uniformemente" ao longo do tempo segundo uma função matemática. Além de poder ser usada na avaliação de projetos de investimentos, a capitalização contínua também pode ser usada na avaliação de opções, derivativos, geração de lucros da empresa, desgaste de equipamentos, entre outras. Na prática, muitas situações exigem o uso da capitalização contínua.

Em tempo contínuo, o período de tempo tende a zero e esse modelo estocástico pode ser representado pela Equação 1.

$$
d \ln V=v d t+\sigma d z
$$

Sendo: $d z$ incremento de Wiener $(d z=\varepsilon \sqrt{d t})$

A suposição de que $\ln V$ tem distribuição normal, implica que a distribuição do valor do projeto em qualquer ponto é lognormal. Esta conclusão é fundamental para a utilização do Movimento Geométrico Browniano para modelar o preço de ações, taxas de juros e diversas outras variáveis econômicas e financeiras. Assim como os preços de ações, por exemplo, não podem cair abaixo de zero, a distribuição lognormal não possui valores negativos. Sendo assim, as alterações no retorno do projeto ao longo do tempo podem ser modeladas pela Equação 2, conforme destacado por Pinto, Brandão e Alves (2009).

$$
d V=\mu V d t+\sigma V d z
$$

Em que:

$\mu$ é taxa drift anual contínua e é dado pela média da série $\left.\ln \left(\mathrm{V}_{\mathrm{i}+1} / \mathrm{V}_{\mathrm{i}}\right)\right)$ somado a $\sigma^{2} / 2$.

Brandão et al. (2005a) comentam sobre o uso do MGB, que, segundo estes autores, é uma ideia relativamente simples, pois se o valor do projeto é assumido para seguir um MGB, então a estimativa deste valor em qualquer ponto no tempo segue uma distribuição lognormal.

A base do teorema proposto por Paul Samuelson (1965) é o MGB; segundo ele, a taxa de retorno de qualquer título seguirá um caminho aleatório seja qual for o padrão do fluxo de caixa que se espera que seja gerado, desde que os investidores tenham informações completas sobre esses fluxos de caixa.

A aplicabilidade deste teorema na estimativa de volatilidade é de grande utilidade, pois, se apenas eventos aleatórios podem fazer com que haja desvio na trajetória dos preços, pode-se então combinar as demais incertezas, tais como: incertezas de preços, de demanda, de custos, em uma única incerteza, a volatilidade do projeto.

\subsection{Abordagem Consolidada da Incerteza de Copeland e Antikarov (2001)}

Pouco tem sido escrito sobre os problemas de estimativa de volatilidade, exceto quanto ao fato de a volatilidade não ser o mesmo que a volatilidade de quaisquer das variáveis-insumo (como preço ou quantidade de produto), nem é igual à volatilidade do patrimônio da empresa. (COPELAND; ANTIKAROV, 2001, p. 245)

O trabalho desenvolvido por Copeland e Antikarov (2001) foi denominado abordagem consolidada da incerteza, pois, segundo os autores, o resultado final é uma única estimativa de incerteza, gerada por meio de diversas incertezas de entrada, tais como: preço, quantidade e custos variáveis. Esta incerteza final é denominada como sendo a variação percentual do valor presente do projeto ao longo do tempo, ou seja, o retorno do projeto.

Na abordagem apresentada, Copeland e Antikarov (2001) se baseiam na suposição de que o valor presente dos fluxos de caixa do projeto sem considerar os investimentos e, ainda, sem flexibilidade, ou seja, sem as opções, é a melhor estimativa do valor de 
mercado do projeto. Com esta consideração, este valor presente é considerado como seu preço de mercado, como se este fosse um ativo negociável.

Fazendo uma analogia da abordagem consolidada da volatilidade com o mercado de ações, podemos dizer que a soma do valor presente dos fluxos de caixa sem investimentos na data zero (valor econômico do ativo) é análogo ao preço da ação na data zero, que é um valor conhecido. O preço futuro da ação, que é um valor desconhecido, pode ser comparado ao valor presente dos fluxos de caixa na data 1 , que também é um valor desconhecido. Sendo assim, um investimento pode ser avaliado como se fosse um ativo negociado no mercado, mesmo que este não seja cotado.

A base do trabalho de Copeland e Antikarov (2001) é o teorema de Paul Samuelson, que prova que a taxa de retorno de um título segue um caminho aleatório, independente do padrão dos fluxos de caixa que são gerados no futuro, ou seja, o valor atual de um título já reflete todas as informações que estão contidas na sua sequência histórica. Esta afirmativa implica dizer que qualquer desvio na trajetória do fluxo de caixa futuro será dado por eventos aleatórios $\mathrm{e}$, consequentemente, os desvios da taxa de retorno também serão aleatórios. Desta forma, os investidores sempre receberão os retornos esperados e o valor da riqueza do projeto irá seguir, com volatilidade constante, de forma aleatória ao longo do tempo. Com base nas ideias de Paul Samuelson, Copeland e Antikarov recorreram ao método de Monte Carlo para combinar diversas incertezas em uma única incerteza, a volatilidade da taxa de retorno.

A Figura 1 ilustra o emprego do método de Monte Carlo para a construção da árvore de eventos, apresentada por Copeland e Antikarov.

De acordo com Pinto, Brandão e Alves (2009), ao se trabalhar com mais de uma variável de entrada (incerteza) em Monte Carlo, deve-se considerar a correlação entre elas na simulação. Considerando, por exemplo, duas variáveis de entrada Preço $(\mathrm{P})$ e
Demanda $(D)$, a correlação entre ambas $\left(\rho_{P D}\right)$ será dada pela correlação entre as séries: $\ln \left(\mathrm{P}_{\mathrm{t}} / \mathrm{P}_{\mathrm{t}-1}\right)$ e $\ln$ $\left(\mathrm{D}_{\mathrm{t}} / \mathrm{D}_{\mathrm{t}-1}\right)$.

A volatilidade da taxa de retorno, apresentada por Copeland e Antikarov (2001), pode ser obtida por meio da Equação 3.

$$
P V_{t}=P V_{0} e^{r t}
$$

Em que (Equação 4):

$$
r t=\ln \frac{P V_{t}}{P V_{0}}
$$

$\mathrm{O}$ valor dos fluxos de caixa futuros é estimado para duas datas, um para a data zero e outro para a data 1. Como a taxa de retorno, ao longo do tempo, é constante, fazemos então $t=1$. Deste modo, a variação percentual do valor do projeto de um período para o seguinte pode ser calculado pela seguinte proporção logarítmica, conforme Equação 5.

$$
z=\ln \frac{P V_{1}+F C F_{1}}{P V_{0}}
$$

Em que: $\mathrm{PV}_{1}$ : valor presente do projeto na data $1 ; \mathrm{FCF}_{1}$ : fluxo de caixa livre na data $1 ; \mathrm{PV}_{0}$ : valor presente do projeto na data zero.

$\mathrm{O}$ valor presente do projeto na data zero e na data 1 pode ser calculado por meio das Equações 6 e 7 , respectivamente.

$$
\begin{gathered}
P V_{0}=\sum_{t=0}^{T} \frac{F C_{t}}{(1+W A C C)^{t}} \\
P V_{1}=\sum_{t=1}^{T} \frac{F C_{t}}{(1+W A C C)^{t-1}}
\end{gathered}
$$

A distribuição de probabilidades dos valores de " $z$ " é obtida pela simulação de Monte Carlo. Mantendo-se o denominador da Equação $5\left(\mathrm{PV}_{0}\right)$ constante e deixando variar o numerador da mesma equação de

\section{Entradas}

Monte Carlo

Ano 1, ano 2

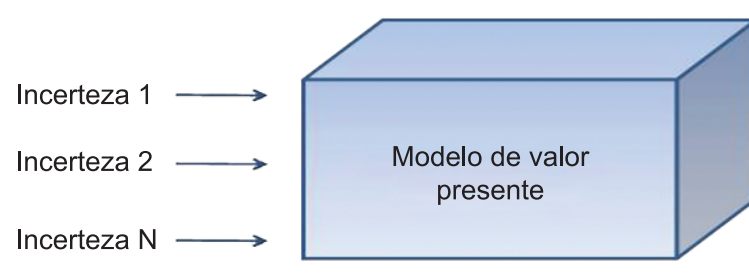

Saídas

Probabilidade do VP

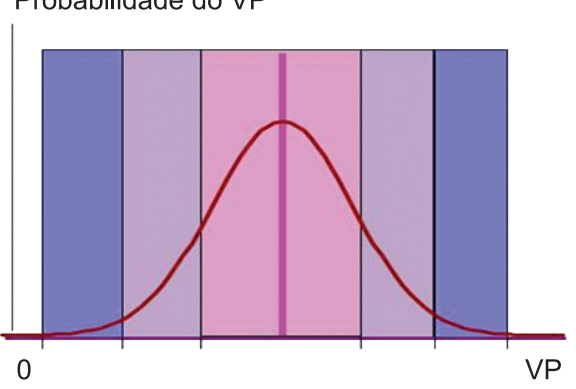

Figura 1. Emprego do método de Monte Carlo para montagem da árvore de eventos. Fonte: Copeland e Antikarov (2001). 
acordo com as incertezas consideradas no projeto, a volatilidade do projeto $(\sigma)$ é então definida como sendo o desvio padrão de "z" $(\sigma=$ desv.pad $(\mathrm{z}))$.

\subsection{A Estimativa de Volatilidade de Herath e Park (2002)}

$\mathrm{Na}$ implementação dos modelos de opções reais, estimar o parâmetro de volatilidade para o ativo subjacente é muito difícil, pois os dados de mercado raramente são disponíveis. (HERATH; PARK, 2002, p. 12)

A estimativa de volatilidade apresentado por Herath e Park (2002), assim como a estimativa de volatilidade apresentada por Copeland e Antikarov (2001), faz uma relação entre o fluxo de caixa na data 1 do projeto e o fluxo de caixa na data zero. Assim a taxa de retorno do projeto é obtida por meio do cálculo do logaritmo Neperiano da soma do fluxo de caixa na data $1 \mathrm{com}$ o fluxo de caixa livre na data 1 , divididos pelo fluxo de caixa na data zero. Por meio de uma simulação de Monte Carlo, simulam-se as diversas incertezas do projeto, chegando-se a uma única incerteza, que será a volatilidade do projeto. As Equações 8, 9 e 10 são apresentadas pelos autores para o cálculo da volatilidade.

$$
\begin{gathered}
\hat{k}=\ln \left(\frac{M V_{1}+A_{1}}{M V_{0}}\right) \\
M V_{1}=\sum_{t=2}^{T} \frac{A_{t}}{(1+k)^{t-1}} \\
M V_{0}=\sum_{t=1}^{T} \frac{A_{t}}{(1+k)^{t}}
\end{gathered}
$$

Em que: $\hat{k}$ : variável aleatória da taxa de retorno do investimento $\left(\hat{k} \sim \mathrm{N}\left(\mu_{\mathrm{k}}, \sigma^{2}\right)\right) ; \mathrm{MV}_{1}$, valor presente dos futuros fluxos de caixa na data $1 ; \mathrm{A}_{1}$ : fluxo de caixa da data $1 ; \mathrm{MV}_{0}$ : valor presente dos futuros fluxos de caixa na data zero; $A_{t}$ : fluxo de caixa da data $\mathrm{t}$; $\mathrm{k}$ : taxa de retorno do investimento.

Comparando-se o método de estimativa de volatilidade apresentado por Herath e Park (2002) com o método de estimativa de volatilidade apresentado por Copeland e Antikarov (2001), podemos destacar algumas diferenças entre ambos, o Quadro 1 destaca a simbologia usada em cada trabalho.

Todavia, a grande diferença entre o método apresentado por Copeland e Antikarov (2001) e o método apresentado por Herath e Park (2002) está no tratamento do valor presente dos futuros fluxos de caixa na data zero, o qual é chamado de $\mathrm{PV}_{0}$ e $\mathrm{MV}_{1}$, respectivamente pelos autores, e é o denominador das Equações 5 e 8. Durante a execução da simulação de
Quadro 1. Comparativo entre a simbologia de Copeland e Antikarov (2001) e Herath e Park (2002).

\begin{tabular}{|l|c|c|}
\hline \multicolumn{1}{|c|}{ Variáveis } & $\begin{array}{c}\text { Copeland e } \\
\text { Antikarov } \\
\mathbf{( 2 0 0 1 )}\end{array}$ & $\begin{array}{c}\text { Herath } \\
\text { e Park } \\
\mathbf{( 2 0 0 2 )}\end{array}$ \\
\hline Valor presente na data zero & $\mathrm{PV}_{0}$ & $\mathrm{MV}_{0}$ \\
\hline Valor presente na data 1 & $\mathrm{PV}_{1}$ & $\mathrm{MV}_{1}$ \\
\hline Fluxo de caixa livre na data 1 & $\mathrm{FCF}_{1}$ & $\mathrm{~A}_{1}$ \\
\hline Taxa de retorno & $\mathrm{Rt}$ & $\mathrm{K}$ \\
\hline
\end{tabular}

Monte Carlo, enquanto Copeland e Antikarov mantêm o $\mathrm{PV}_{0}$ fixo, sem variar juntamente com as incertezas do projeto, variando somente o $\mathrm{PV}_{1}$; Herath e Park consideram, ambos, os valores presentes dos futuros fluxos de caixa na data zero e na data 1 como variáveis aleatórias independentes e que tanto uma, quanto outra, devem variar juntamente com as incertezas do projeto durante a simulação de Monte Carlo. Esta importante diferença entre as duas abordagens vai gerar valores diferentes para a volatilidade do projeto.

\subsection{A Estimativa da Volatilidade por meio da Dependência de $\mathrm{VP}_{\mathrm{o}}$ e VP $\mathrm{P}_{1}$}

Assumindo algumas propostas dos autores discorridos anteriormente, Copeland e Antikarov (2001), Herath e Park (2002), e propondo algumas mudanças nas suas abordagens, neste capítulo, é proposto um novo modelo para a determinação da volatilidade. Ao final do estudo, este modelo proposto será comparado, por meio de um caso real, com os demais modelos.

As propostas dos autores discorridos anteriormente que serão absorvidas por esta nova abordagem são as seguintes:

i. A Negativa do Ativo Negociado (Market Asset Disclaimer - MAD), que assume que o VPL, sem opções, é a melhor alternativa para o preço de mercado do projeto. Isto garante que o retorno esperado do projeto será exatamente o mesmo que a taxa de desconto ajustada ao risco, desde que o mercado seja eficiente, garantindo-se assim um VPL igual a zero;

ii. O valor presente do projeto na data 1 , o fluxo de caixa na data 1 e o valor presente do projeto na data zero serão utilizados como parâmetros de entrada para o cálculo da volatilidade;

iii. O uso da simulação de Monte Carlo para combinar as diversas incertezas do projeto em uma única incerteza, a volatilidade do projeto.

Esta nova abordagem propõe, no entanto, um novo tratamento do valor presente do projeto na data zero durante a simulação de Monte Carlo. Copeland e Antikarov (2001) tratam o valor presente do projeto 
na data zero $\left(\mathrm{PV}_{\mathrm{o}}\right)$ como sendo fixo, ou seja, ele não varia durante a simulação. Herath e Park (2002) propõem variar o valor presente na data zero, o qual os autores chamam de $\left(\mathrm{MV}_{\mathrm{o}}\right)$, durante a simulação de Monte Carlo, porém de forma independente do valor presente do projeto na data 1 e do fluxo de caixa na data 1. Estas duas abordagens vão resultar em valores diferenciados da volatilidade.

Neste novo modelo, entende-se que o valor presente do projeto na data zero, o qual é denominado $\mathrm{VP}_{\mathrm{o}}$, deve variar, juntamente com o valor presente do projeto na data $1\left(\mathrm{VP}_{1}\right)$, porém de forma dependente um do outro. Uma vez que o valor presente do projeto na data zero $\left(\mathrm{VP}_{\mathrm{o}}\right)$ depende do valor presente do projeto em todas as datas futuras, e a data 1 é uma destas datas futuras, ambos, $\mathrm{VP}_{\mathrm{o}}$ e $\mathrm{VP}_{1}$, devem variar de forma dependente durante a simulação de Monte Carlo.

As Equações 11, 12 e 13 apresentadas para o cálculo desta nova abordagem são semelhantes às apresentadas por Copeland e Antikarov (2001) e por Herath e Park (2002).

$$
\begin{gathered}
z=\ln \frac{V P_{1}+F C L_{1}}{V P_{0}} \\
V P_{0}=\sum_{t=0}^{T} \frac{F C_{t}}{(1+r)^{t}} \\
V P_{1}=\sum_{t=1}^{T} \frac{F C_{t}}{(1+r)^{t-1}}
\end{gathered}
$$

Em que: $\mathrm{VP}_{\mathrm{o}}$ : valor presente do projeto na data zero; $\mathrm{FCL}_{1}$ : fluxo de caixa livre na data $1 ; \mathrm{VP}_{1}$ : valor presente do projeto na data $1 ;$ r: taxa de desconto adequada ao risco.

Calculado o valor presente do projeto na data zero $\left(\mathrm{VP}_{0}\right)$ e o valor presente do projeto na data 1 $\left(\mathrm{VP}_{1}\right)$, conforme Equações 12 e 13, respectivamente, usam-se estes dois parâmetros como dados de entrada da Equação 11.

Por meio da simulação de Monte Carlo, é obtida a distribuição de probabilidades dos valores de " $z$ ". Variando-se o numerador e o denominador da Equação 11 de forma dependente um do outro, de acordo com as incertezas consideradas no projeto, a volatilidade do projeto $(\sigma)$ é então definida como sendo o desvio padrão de "z", conforme Equação 14.

$$
\sigma=\operatorname{desv} \cdot \operatorname{pad}(z)
$$

\section{Modelagem}

A aplicação das abordagens de estimativa da volatilidade de Copeland e Antikarov (2001), Herath e Park (2002) e a Estimativa da Volatilidade por meio da Dependência de $\mathrm{VP}_{\mathrm{o}}$ e $\mathrm{VP}_{1}$, proposta neste trabalho, será feita por meio da modelagem de um projeto real de uma indústria de transformação.

\subsection{O projeto}

Um investimento em uma nova unidade de uma empresa deve ser analisado de forma sistemática e detalhada, não somente para decidir quando o investimento deverá ser realizado, como também inserir na análise as flexibilidades necessárias que a empresa irá necessitar no futuro (TRIANTIS; BORISON, 2001).

O projeto em análise será chamado de Projeto $\mathrm{X}$ e será realizado em três etapas. A primeira etapa consiste no pré-projeto, nele se realizará, entre outros itens, estudos de viabilidade técnica, visitas a fornecedores e cotações diversas; ao final da primeira etapa a empresa tem a opção de continuar com o investimento e realizar a segunda etapa. A segunda etapa consiste em toda obra civil necessária para a implantação da nova unidade. Por obra civil entende-se a construção do galpão e toda infraestrutura necessária para seu funcionamento. A terceira etapa consiste na compra e instalação dos equipamentos fabris, que a princípio irão produzir dois produtos, aqui chamados de "Produto A" e "Produto B".

Concluída a segunda etapa, a empresa tem a opção de dar sequência no projeto e realizar também a terceira etapa. Se a empresa desistir de realizar

Tabela 1. Dados do projeto.

\begin{tabular}{lr}
\hline \multicolumn{1}{c}{ Parâmetros } & \multicolumn{1}{c}{ Valores } \\
\hline Taxa de Desconto Adequada ao Risco & $13 \%$ \\
Investimento Inicial - Data zero (USD) & 75,000 \\
Investimento no Galpão - Data 1 (USD) & 318,181 \\
Investimento em Equipam - Data 2 (USD) & $2,368,181$ \\
Depreciação anual & $12.5 \%$ \\
Custos Produto A (\% Preço em USD) & $77.8 \%$ \\
Custos Produto B (\% Preço em USD) & $51.5 \%$ \\
IR & $34 \%$ \\
\hline
\end{tabular}

Tabela 2. Dados dos produtos.

\begin{tabular}{ccccc}
\hline Ano & \multicolumn{2}{c}{ Produto A } & \multicolumn{2}{c}{ Produto B } \\
\cline { 2 - 5 } & $\begin{array}{c}\text { Preço } \\
\text { Unitário } \\
\text { (USD) }\end{array}$ & $\begin{array}{c}\text { Demanda } \\
\left(\mathbf{m}^{2}\right)\end{array}$ & $\begin{array}{c}\text { Preço } \\
\text { Unitário } \\
\text { (USD) }\end{array}$ & $\begin{array}{c}\text { Demanda } \\
\left(\mathbf{m}^{2}\right)\end{array}$ \\
\hline 1 & 28.55 & 12,650 & 12.55 & 110,870 \\
2 & 28.69 & 17,875 & 12.68 & 156,658 \\
3 & 28.57 & 24,908 & 12.76 & 202,087 \\
4 & 28.57 & 26,153 & 13.02 & 237,483 \\
5 & 28.57 & 27,461 & 13.28 & 254,357 \\
6 & 28.57 & 28,834 & 13.54 & 252,984 \\
7 & 28.57 & 30,276 & 13.81 & 251,542 \\
8 & 28.57 & 30,276 & 14.09 & 251,542 \\
9 & 28.57 & 30,276 & 14.37 & 251,542 \\
10 & 28.57 & 30,276 & 14.66 & 251,542 \\
\hline
\end{tabular}


a terceira etapa, ela poderá utilizar o galpão para armazenagem. Nesse caso, a empresa não irá produzir aqui no Brasil e irá importar os produtos A e B. A empresa tem, nas mãos, duas opções de compra. Uma pode ser exercida a qualquer momento após a conclusão da primeira etapa do projeto, e a outra também poderá ser exercida a qualquer momento, porém ao final da segunda etapa.

Para a empresa calcular o valor das opções de compra que tem nas mãos, será necessário conhecer a volatilidade do projeto.

A escolha deste objeto de estudo se justifica, no presente trabalho, não somente pela relevância da flexibilidade da variedade de produtos que poderão ser comercializados por esta nova unidade, bem como pela importância mundial da empresa no setor em que está inserida, o que pressupõe uma gestão econômico-financeira como atividade estratégica.

\subsubsection{Dados do projeto}

Os dados do Projeto X para o cálculo do Fluxo de Caixa Descontado (Cálculo Tradicional) são apresentados na Tabela 1.

Ressalta-se que os valores apresentados nas Tabelas 1 e 2 foram devidamente alterados para

Tabela 3. Fluxo de Caixa Descontado.

\begin{tabular}{|c|c|c|c|}
\hline Ano & $\begin{array}{c}\text { FC depois do IR } \\
\text { (USD) }\end{array}$ & \multicolumn{2}{|c|}{$\begin{array}{l}\text { VP dos fluxos de Caixa } \\
\text { (USD) }\end{array}$} \\
\hline 0 & $-159,960$ & $\mathrm{VP}_{0}$ & $2,732,715$ \\
\hline 1 & 82,492 & $\mathrm{VP}_{1}$ & $3,268,723$ \\
\hline 2 & 304,523 & $\mathrm{VP}_{2}$ & $3,600,440$ \\
\hline 3 & 439,817 & $\mathrm{VP}_{3}$ & $3,724,386$ \\
\hline 4 & 600,581 & $\mathrm{VP}_{4}$ & $3,711,563$ \\
\hline 5 & 714,478 & $\mathrm{VP}_{5}^{4}$ & $3,515,410$ \\
\hline 6 & 761,860 & $\mathrm{VP}_{6}$ & $3,165,053$ \\
\hline 7 & 791,133 & $\mathrm{VP}_{7}$ & $2,715,608$ \\
\hline 8 & 823,127 & $\mathrm{VP}_{8}$ & $2,174,657$ \\
\hline 9 & 842,038 & $\mathrm{VP}_{9}$ & $1,527,229$ \\
\hline 10 & 774,266 & $\mathrm{VP}_{10}$ & 774,266 \\
\hline
\end{tabular}

garantir a confidencialidade do projeto, todavia o procedimento adotado não compromete o resultado obtido.

\subsubsection{Fluxo de Caixa Descontado (Avaliação Tradicional)}

A Tabela 3 apresenta, com base em uma Taxa de Desconto Adequada ao Risco de $13 \%$, os fluxos de caixa após o Imposto de Renda e o valor presente dos fluxos de caixa a cada período.

Descontando-se o investimento realizado na data 2 e na data 1 a uma Taxa de Desconto Adequada ao Risco de $13 \%$, somado ao investimento realizado na data zero, mostrados na Tabela 1 , chega-se a um valor presente do investimento na data zero de USD $2,211,211$. Com esse valor de investimento na data zero tem-se um Valor Presente Líquido (VPL) do projeto de USD 521,504 (USD 2,211,211 - USD $2,732,715)$. Pela análise tradicional, com um VPL positivo, o projeto é considerado viável e deve ser incentivada a sua realização.

\subsubsection{Aplicação das diversas abordagens da volatilidade}

A Abordagem Consolidada da Volatilidade de Copeland e Antikarov (2001), a Estimativa de Volatilidade de Herath e Park (2002) e a Estimativa da Volatilidade por meio da Dependência de VPo e VP1, proposta por este trabalho, apresentadas no capítulo 2, serão aplicadas separadamente no cálculo da volatilidade do Projeto X.

A variável estocástica escolhida para a simulação de Monte Carlo foi o preço dos produtos (Produto A e Produto B) a serem fabricados na unidade sob estudo. $\mathrm{O}$ preço, por não poder assumir valores negativos, segue uma distribuição lognormal.

No Quadro 2, são apresentadas as equações e as principais considerações de cada autor para o cálculo da volatilidade.

Quadro 2. Equações e considerações sobre a volatilidade.

\begin{tabular}{|c|c|c|}
\hline Autor & $\begin{array}{c}\text { Equação p/ cálculo da } \\
\text { Volatilidade }\end{array}$ & Considerações \\
\hline $\begin{array}{c}\text { Copeland e } \\
\text { Antikarov }\end{array}$ & $z=\ln \frac{P V_{1}+F C F_{1}}{P V_{0}}$ & $\begin{array}{c}\text { Na simulação de Monte Carlo, VP deve ser mantido fixo, } \\
\mathrm{VP}_{1} \text { e FCF } \mathrm{FC}_{1} \text { devem variar. }\end{array}$ \\
\hline $\begin{array}{c}\text { Herath e } \\
\text { Park }\end{array}$ & $\hat{k}=\ln \left(\frac{M V_{1}+A_{1}}{M V_{0}}\right)$ & $\begin{array}{c}\mathrm{MV}_{1} \text { e } \mathrm{MV}_{\mathrm{o}} \text { são consideradas variáveis aleatórias } \\
\text { independentes e tanto uma quanto a outra devem } \\
\text { variar na simulação de Monte Carlo. }\end{array}$ \\
\hline $\begin{array}{c}\text { Modelo } \\
\text { Proposto }\end{array}$ & $z=\ln \frac{V P_{1}+F C L_{1}}{V P_{0}}$ & $\begin{array}{c}\text { Tanto VP } \mathrm{VP}_{1} \text { quanto VP devem variar na simulação de } \\
\text { Monte Carlo, porém de forma dependente. }\end{array}$ \\
\hline
\end{tabular}




\begin{tabular}{|c|c|c|}
\hline \multicolumn{2}{|c|}{ C forecast: $z$} & - \\
\hline & View Forecast Prefer & \\
\hline \multicolumn{2}{|c|}{10.000 Trials } & 9.899 Displayed \\
\hline & Statistic & Forecast values \\
\hline \multirow[t]{4}{*}{ - } & Trials & 10.000 \\
\hline & Mean & 0,13 \\
\hline & Median & 0.17 \\
\hline & Mode & ... \\
\hline & Standard Deviation & 0,33 \\
\hline & Variance & 0.11 \\
\hline & Skewness & -0.9371 \\
\hline & Kuttosis & 5,87 \\
\hline & Coeff. of Variability & 2,46 \\
\hline & Minimum & $-3,19$ \\
\hline & Maximum & 1,03 \\
\hline & Mean Std. Error & 0,00 \\
\hline
\end{tabular}

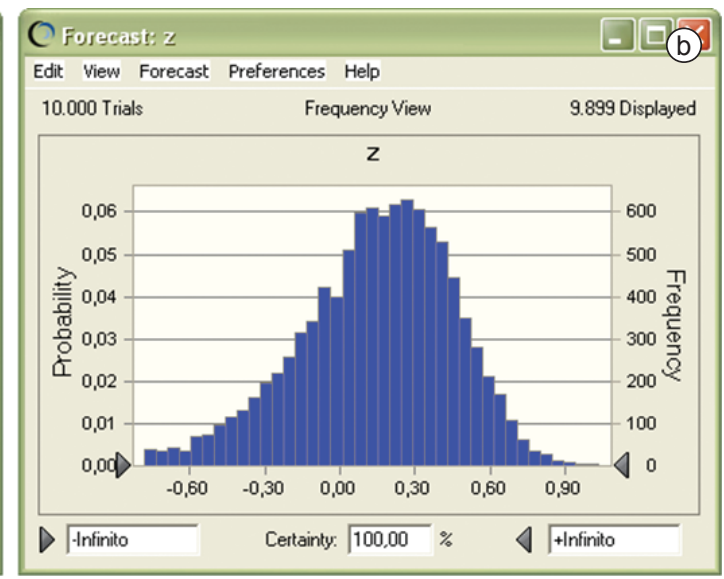

Figura 2. Análise de Monte Carlo para a abordagem de volatilidade de Copeland e Antikarov (2001).

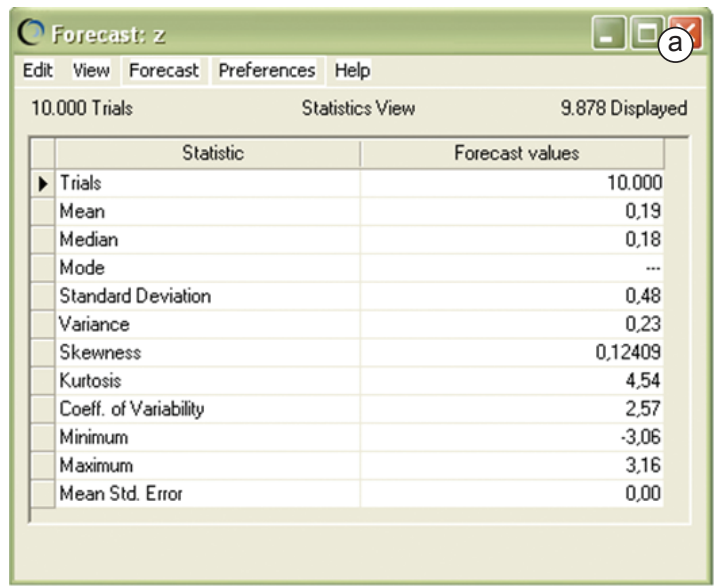

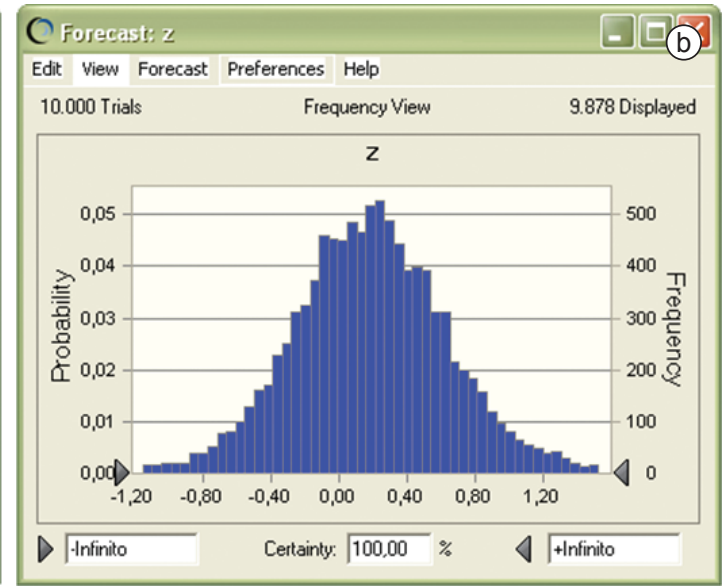

Figura 3. Análise de Monte Carlo para a abordagem de volatilidade de Herath e Park (2002).

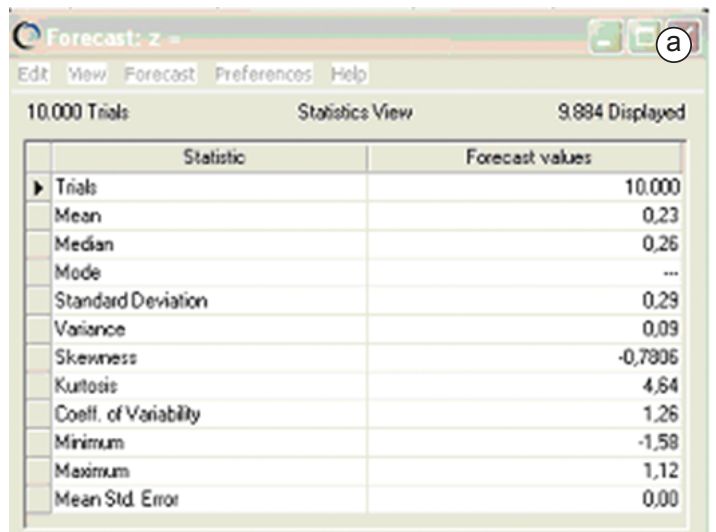

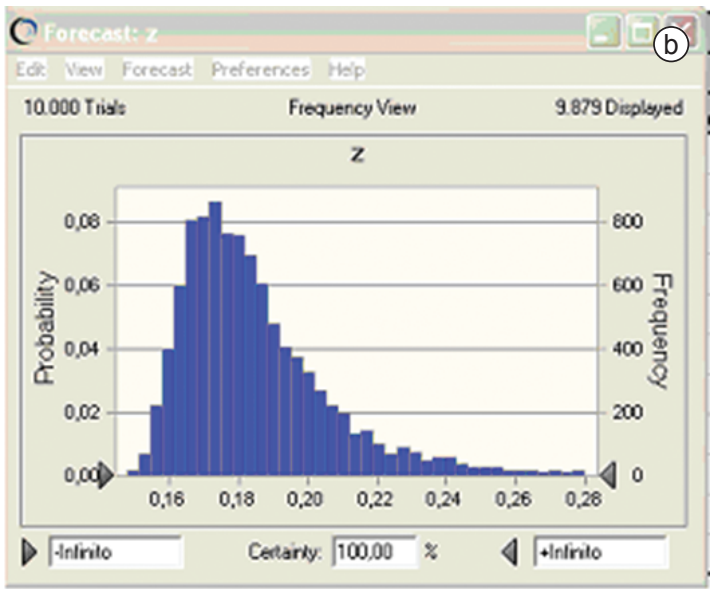

Figura 4. Análise de Monte Carlo para a abordagem de volatilidade do modelo proposto.

Foi realizada uma rodada de simulação de Monte Carlo para cada uma das três abordagens da volatilidade apresentadas, com 10.000 iterações cada uma. A análise de sensibilidade necessária para a estimativa da volatilidade foi processada no programa Crystal Ball ${ }^{\circledR}$. Os resultados são apresentados nas Figuras 2-4 e no Quadro 3.
Quadro 3. Resultados.

\begin{tabular}{|l|c|c|}
\hline \multicolumn{1}{|c|}{ Autor } & $\begin{array}{c}\mathbf{Z} \\
(\mathbf{\%})\end{array}$ & $\begin{array}{c}\text { Volatilidade } \\
(\mathbf{\%})\end{array}$ \\
\hline Copeland e Antikarov & 17,91 & 33 \\
\hline Herath e Park & 17,91 & 48 \\
\hline Modelo Proposto & 17,91 & 29 \\
\hline
\end{tabular}




\section{Conclusões}

A pesquisa na área de opções reais e particularmente no cálculo da volatilidade tem experimentado um contínuo progresso nas últimas décadas. Seguindo esta vereda, este trabalho propôs apresentar alguns modelos concebidos por meio de revisão bibliográfica para o cálculo da volatilidade, assim como a proposta de uma nova sistemática.

A apresentação desta nova sistemática, conforme destacado no objetivo, foi apenas para contribuir com a discussão do cálculo da volatilidade, não se teve aqui a intenção de provar que esta nova sistemática seja melhor que as sistemáticas já existentes.

Fazendo-se um comparativo entre a sistemática de determinação da volatilidade proposta por Copeland e Antikarov (2001), a sistemática proposta por Herath e Park (2002) e a sistemática proposta por este trabalho, percebe-se que o fato de se considerar ou não como fixo o valor presente do projeto sob estudo na data zero, ou de se considerar o valor presente na data zero dependente ou independente do valor presente na data 1, durante a simulação de Monte Carlo, gera valores simulados bem distintos de volatilidade do projeto.

Conforme apresentado no Quadro 3, verifica-se que o valor calculado da volatilidade utilizando a abordagem de Copeland e Antikarov (2001) foi de 33\% para o projeto sob estudo, enquanto que o valor calculado utilizando-se a abordagem de Herath e Park (2002) foi de $48 \%$ e do modelo proposto por este trabalho foi de $29 \%$, apesar de, nas três abordagens, o valor de " $z$ " ser o mesmo.

Percebe-se que há uma diferença de 19 pontos percentuais entre a menor volatilidade encontrada (Modelo proposto por este trabalho) e a maior volatilidade encontrada (Abordagem de Herath e Park (2002)), sendo a volatilidade calculada sob a abordagem proposta por Herath e Park (2002) 65,5\% maior que a volatilidade calculada sob o modelo proposto por este trabalho.

Diante do comparativo acima, conclui-se que a determinação da volatilidade em projetos industriais para a análise de risco em investimentos ainda é um parâmetro em que não há uma convergência final sob sua forma de ser determinada. Embora haja consenso em alguns aspectos, como a consideração de que o valor do projeto na data zero, sem a opção, é a melhor estimativa para o valor de mercado do projeto e que a volatilidade é dada como o desvio padrão da variável "z", ainda assim há uma diferença considerável de valores entre as três abordagens aplicadas no projeto sob estudo, diferença esta que pode comprometer a decisão ou não de se investir no projeto.

As decisões estratégicas de uma empresa devem visar a sobrevivência a longo prazo pela criação de sustentabilidade no meio em que a empresa está inserida, assim sendo, um outro aspecto de contribuição deste trabalho é a diminuição da ponte entre o meio acadêmico e o meio industrial, uma vez que um assunto já bastante difundido no meio acadêmico foi aplicado em um projeto industrial, no qual ainda há carência no seu conhecimento.

A aplicação da metodologia e os resultados apresentados são preliminares, uma vez que outras abordagens de volatilidade poderão ser incorporadas ao presente trabalho, abrindo um leque para um comparativo maior entre as diversas sistemáticas propostas, buscando assim uma convergência ou não entre os diversos modelos.

\section{Referências}

BERTRAND, J.; FRANSOO, J. Modelling and Simulation Operations management research methodologies using quantitative modeling. International Journal of Operations \& Production Management, v. 22, n. 2, p. 241-264, 2002. http://dx.doi.org/10.1108/01443570210414338

BLACK, F.; SCHOLES, M. The pricing of options and corporate liabilities. Journal of Political Economy, v. 81, p. 637-659, 1973. http://dx.doi. org/10.1086/260062

BRANDÃO, L.; DYER, J.; HAHN, W. Using binomial decision trees to solve real-option valuation problems. Decision Analysis, v. 2, n. 2, p. 69-88, 2005a. http:// dx.doi.org/10.1287/deca.1050.0040

BRANDÃO, L.; DYER, J.; HAHN, W. Response to Comments on Brandão et al. Decision Analysis, v. 2, n. 2, p. 103-109, 2005b.

COPELAND, T.; ANTIKAROV, V. Opções reais: um novo paradigma para reinventar a avaliação de investimentos. Editora Campus, 2001.

HERATH, H.; PARK, C. Multi-stage capital investiment opportunities as compound real options. The Engineering Economist, v. 47, n. 1, p. 1-27, 2002.

LUEHRMAN, T. Investiment opportunities as real options: getting started on the numbers. Harvard Business Review, p. 51-67, 1998.

MIRANDA FILHO, J. Investimento em distribuição utilizando opções Reais. 2005. 160 f. Dissertação (Mestrado Ciências em Engenharia Elétrica)-Universidade Federal de Itajubá, Itajubá, 2005.

PINTO, C. B.; BRANDÃO, L.; ALVES, M. L. Valuing the switching flexibility of the ethanol-gas flex fuel. Annals of Operations Research, v. 176, n. 1, p. 333-348, 2009.

SAMUELSON, P. Proof that properly anticipated prices fluctuate randomly. Industrial Management Review, v. 6, p. 41-49, 1965.

SANTOS, E.; PAMPLONA, E. Teoria das Opções Reais: uma atraente opção no processo de análise de investimentos. Revista de Administração da Universidade de São Paulo, v. 40, n. 3, 2005.

SMITH, J. Alternative approachs for solving real options problems. (Coments on Brandão et al. 2005). Decision Analysis, v. 2, p. 89-102, 2005. http://dx.doi.org/10.1287/ deca.1050.0041

TRIANTIS, A; BORISON, A. Real Options: state of the practice. Journal of Applied Corporate Finance, v. 14.2, 2001. 